\title{
Stimulation of Denervated Rat Soleus Muscle with Fast and Slow Activity Patterns Induces Different Expression of Acetylcholinesterase Molecular Forms ${ }^{1}$
}

\author{
TERJE LQMO, ${ }^{2}$ JEAN MASSOULIÉ,* AND MARC VIGNY*
}

Institute of Neurophysiology, University of Oslo, Oslo 1, Norway and *Laboratoire de Neurobiologie, École Normale Supérieure, 75230 Paris, France

\begin{abstract}
The relative amount and distribution of acetylcholinesterase (AChE) molecular forms were studied in slow soleus and (less extensively) in fast extensor digitorum longus (EDL) muscles of the rat before and after denervation and direct stimulation. Normal EDL muscles showed higher total and specific AChE activity than normal soleus muscles and contained essentially three different molecular $\mathbf{A C h E}$ forms $\left(G_{1}\right.$, $G_{4}$, and $\left.A_{12}\right)$ as opposed to six forms $\left(G_{1}, G_{2}, G_{4}, A_{4}, A_{8}\right.$, and $\left.A_{12}\right)$ in the soleus. Denervation reduced $A C h E$ activity in both muscles. In the soleus direct stimulation starting 2 to 3 weeks after denervation increased the specific AChE activity markedly. The increase started 12 to $24 \mathrm{hr}$ after the onset of stimulation, reached 3 to 5 times normal values after 2 to 7 days, and then declined gradually toward normal values over the next 2 weeks. Furthermore, the effect on the different molecular forms depended strongly on the stimulus pattern. Thus, intermittent $100 \mathrm{~Hz}$ stimulation (fast pattern) induced essentially the three forms typical of the normal EDL, whereas continuous $10 \mathrm{~Hz}$ stimulation induced the six forms characteristic of normal soleus muscles but with some differences in their relative proportions. In the EDL, 2 days of continuous $10 \mathrm{~Hz}$ stimulation (the only duration and pattern examined) failed to induce a similar increase in AChE activity.
\end{abstract}

Acetylcholinesterase (AChE) plays an essential role in cholinergic transmission, particularly at the neuromuscular junction, by rapidly hydrolysing acetylcholine ( $\mathrm{ACh}$ ) after its release into the synaptic cleft from nerve terminals (Katz and Miledi, 1973). AChE exists in a number of molecular forms which may be classified as globular forms (monomers $G_{1}$, dimers $G_{2}$, and tetramers $G_{4}$ ) and asymmetric or collagen-tailed forms which consist of assemblies of one, two, or three tetramers $\left(A_{4}, A_{8}\right.$, and $\left.A_{12}\right)$ with a collagen-like element (Massoulie and $B o n, 1982)$. The $A$-forms have received considerable attention because of suggestions that they play a special role in nerve-muscle interaction. These forms are present along the entire length of some embryonic and neonatal muscles (Sketelj and Brzin,

Received June 11, 1984; Revised October 17, 1984;

Accepted October 24, 1984

\footnotetext{
${ }^{1}$ This work was supported by The Muscular Dystrophy Association of America and The Norwegian Research Council for Science and the Humanities. We thank Mr. P. Allemand and Mrs. Sigrid Schaller for skilled technical assistance.

'To whom correspondence should be addressed, at Institute of Neuro-
} physiology, University of Oslo, Karl Johansgate. 47, 0162. Oslo 1, Norway.
1980; Koenig and Rieger, 1981) but are highly concentrated at the endplate of some adult rat muscles where they essentially disappear after denervation and reappear following reinnervation (Hall, 1973; Vigny et al., 1976; Collins and Younkin, 1982; Younkin et al. 1982). More recently, however, the A-forms have been detected also outside the endplate in adult human muscles (Carson et al., 1979), and they have also been reported to increase markedly after denervation in a rabbit slow muscle (Bacou et al., 1982).

Our present understanding of the mechanisms underlying the regulation of AChE in muscles is limited. Several factors must be considered. First, early contact with the motor nerve appears to play an important role by leaving permanent instructions for later synthesis and localization of AChE at the endplate (Koenig and Vigny, 1978; L $\phi \mathrm{mo}$ and Slater, 1980; Weinberg and Hall, 1979). Second, muscle activity probably plays an essential role in the control of AChE because it has been shown that AChE activity falls after suppression of muscle activity by tetrodotoxin (Rieger et al., 1980; Brockman et al., 1984), blockage of neuromuscular transmission (Drachman, 1972; Rubin et al., 1980), or neural conduction (Butler et al., 1978; Cangiano et al., 1980). Conversely, AChE may be induced to appear at denervated ectopic endplates by direct muscle stimulation ( $L \phi \mathrm{mo}$ and Slater, 1980). Third, there is strong evidence that substances released from motor nerve terminals contribute to the regulation of AChE at the neuromuscular junction. Thus, soluble extracts from motor nerves partly prevent the denervation-induced decay of $A C h E$ activity, including the $A_{12}$ forms in rat muscles maintained in organ culture (Davey et al., 1979; Fernandez et al., 1980). Thosc substances appear to be carried by axonal transport and are released in increased amounts when the nerve is stimulated (Davey et al., 1979).

In the present experiments we have abolished all evoked muscle activity and removed all direct neurotrophic influences by denervating the fast extensor digitorum longus (EDL) and slow soleus (SOL) muscles of the rat. After 2 to 3 weeks of denervation, when AChE content is low, we have started chronic stimulation of the muscles to examine the effect of evoked muscle activity on muscle AChE in the absence of direct neural influences. Further information was obtained by using two different patterns of stimulation: one resembling the impulse pattern normally present in a fast muscle such as the rat EDL (fast pattern) and one resembling the pattern in a normal slow muscle, such as the SOL (slow pattern) (Fischbach and Robbins, 1969; Hennig and $L \phi m o, 1984)$. Recent work shows that the distribution of AChE molecular forms is different in rat EDL and SOL muscles (Gisiger and Stephens, 1982; Groswald and Dettbarn, 1983), and it is possible that this is related to the different firing patterns in fast and slow motor units. In this connection it is interesting that slow muscles become fast during stimulation with a fast stimulus pattern, whereas fast muscles become slow during stimulation with a slow stimulus pattern ( $L \phi m o$ et al., 1974; Salmons 
and Sreter, 1976). We were therefore interested in whether muscles show similar impulse paltern-dependent plasticity with respect to their content of different AChE molecular forms. If so, this may throw new light not only on the role of muscle activity in AChE regulation but also on the function of the different molecular forms.

\section{Materials and Methods}

Denervation and chronic stimulation. Under ether anesthesia a 5 to 10 mm-long segment of the sciatic nerve was removed from the thigh on one or both sides of young male Wistar rats weighing 200 to $250 \mathrm{gm}$. Ten to 15 days later, two Teflon-coated multistranded steel wires (outer diameter, 0.011 $\mathrm{mm}, \mathrm{AS} 632$, Cooner Sales, Chatsworth, CA) were implanted into the right leg under anesthesia (Equithesin, $0.4 \mathrm{ml} / 100 \mathrm{gm}$ of body weight). The distal ends of the wires with the insulation removed were placed across the SOL or EDL, one end proximally and posteriorly and the other end distally and anteriorly. Both wires were run under the skin to the head and into flexible silicon tubes (outer diameter, $6 \mathrm{~mm}$ ) which were fixed to the skull with screws and dental cement. The wires and the protecting tubes were connected to a rotating contact approximately $1 \mathrm{~m}$ above the rat, which moved freely in a wide bucket. Stimulation usually started 2 to 3 weeks after denervation and lasted for $6 \mathrm{hr}$ up to 21 days. The stimulation was either continuous at $10 \mathrm{~Hz}$ (slow pattern) or 60 pulses at $100 \mathrm{~Hz}$ every $60 \mathrm{sec}$ (mean frequency, $1 \mathrm{~Hz}$; fast pattern). Each stimulus was bipolar with a duration of $0.2 \mathrm{msec}$ and an intensity of 5 to $10 \mathrm{~mA}$ in each direction.

Acute experiments. At the end of the stimulation period, the muscles were exposed under ether anesthesia and the nerve trunk was stimulated. No contraction was seen with the dissection microscope, showing that $\mathrm{no}$ reinnervation had occurred, except in two muscles which were not included The muscles were then removed and, in one series of experiments, pinned out in a bath with oxygenated saline. An endplate-containing central region and an endplate-free proximal region of about equal length ( 3 to $4 \mathrm{~mm}$ ) were cut from each muscle and frozen in liquid nitrogen for subsequent biochemical analysis. In two other series, the tendons were trimmed away and the muscles were frozen whole in liquid nitrogen.

Biochemical analysis. The muscles were homogenized in a detergent saline buffer containing anti-proteolytic agents (Tris-HCl, pH 7, 10 mm; EDTA, $10 \mathrm{~mm} ; \mathrm{NaCl}, 1 \mathrm{M}$; Tritor X-100, 1\%; benzanidine, $1 \mathrm{~mm}$; Zymofren (aprotinin) 25 units $/ \mathrm{ml}$; bacitracin, $1 \mathrm{mg} / \mathrm{ml}$ ). The AChE activity was determined by the spectrophotometric method of Ellman et al. (1961), and the proteins were assayed by the method of Lowry et al. (1951) with bovine serum albumin as standard. AChE activities are expressed as nanomoles of acetylthiocholine hydrolyzed per minute at $28^{\circ} \mathrm{C}$, either per muscle or per milligram of protein. Molecular forms of AChE were analyzed by centrifuging an aliquot of the muscle extract in a sucrose gradient ( 5 to $20 \%$ sucrose in $1 \mathrm{M} \mathrm{NaCl}, 50 \mathrm{~mm}$ $\mathrm{MgCl}_{2}, 10 \mathrm{mM}$ Tris $\mathrm{HCl}, \mathrm{pH} 7,1 \mathrm{mg} / \mathrm{ml}$ of bacitracin, $1 \%$ Triton $\mathrm{X}-100$ ) in a Beckman SW 41 rotor at $4^{\circ} \mathrm{C}, 40,000 \mathrm{rpm}$ for $20 \mathrm{hr}$. Sedimentation coefficients were determined by comparison with $\beta$-galactosidase from Escherichia coli (16 S) and alkaline phosphatase from calf intestine (6.1 S).

\section{Results}

AChE in normal and denervated $S O L$ and EDL

Activity. The effect of denervation on SOL muscles was examined in three different series of experiments. In the first two series (Figs.
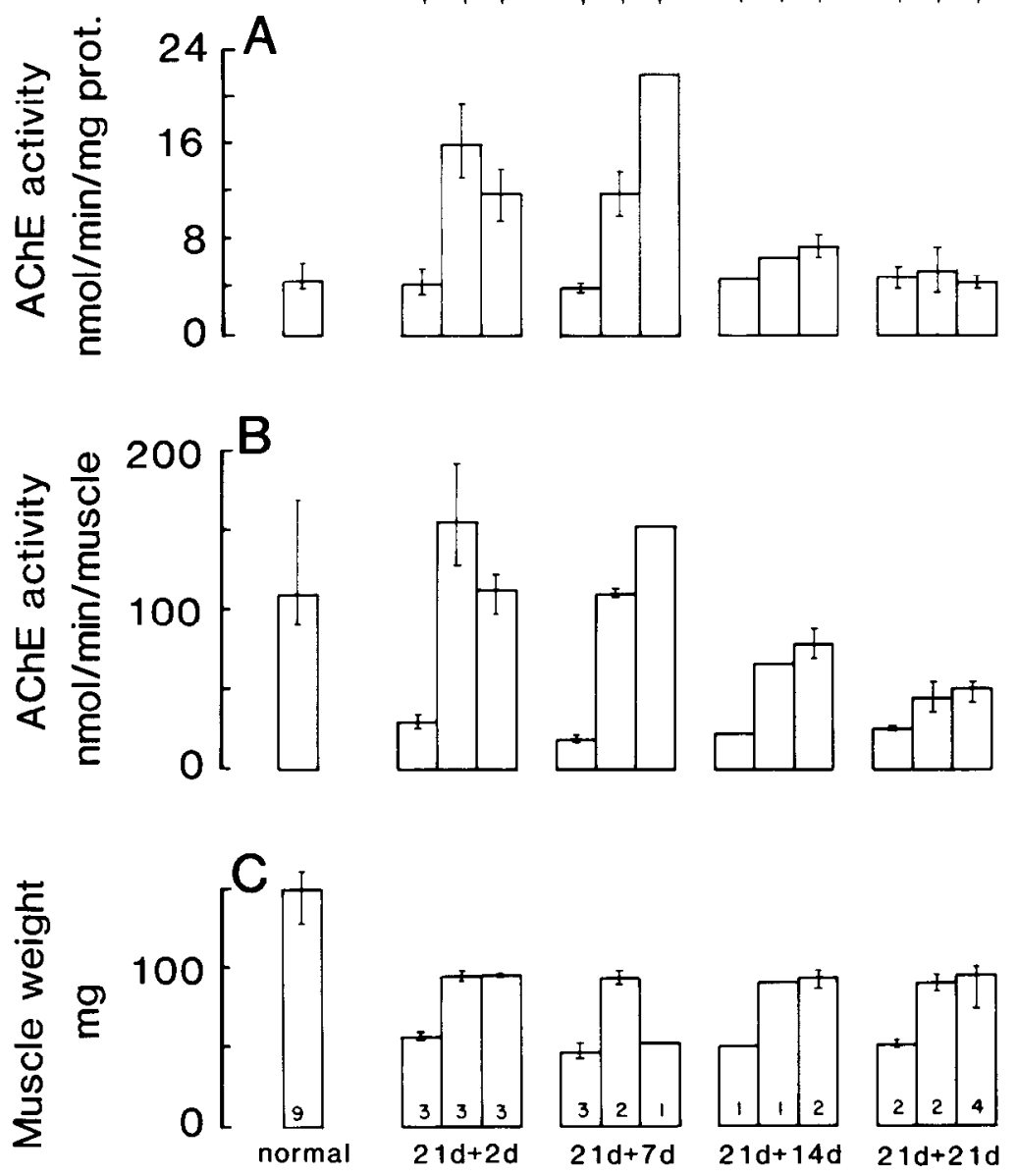

Denervation 23-42 days

Stimulation last $2-21$ days
Figure 1. Stimulation increases specific AChE activity $(A)$, total AChE activity $(B)$, and muscle weight $(C)$ in denervated SOL muscles. Intermittent $100 \mathrm{~Hz}$ or continuous $10 \mathrm{~Hz}$ stimulation (see "Materials and Methods") started 21 days after denervation and lasted $2,7,14$, or 21 days as indicated. For each point in time (group of three bars) arrowheads on top indicate denervation only or denervation + stimulation at 10 or $100 \mathrm{~Hz}$. The height of each bar indicates mean values, the narrow bars give the total range of all observations, and the numbers in the bars give the number of muscles. 

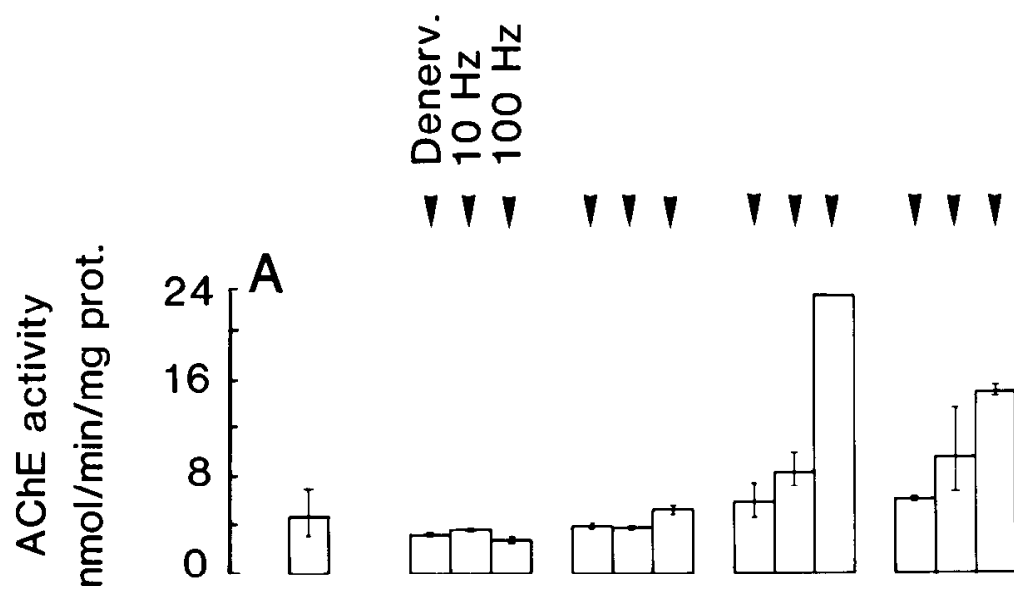

Figure 2. Stimulation increases specific $A C h E$ activity $(A)$, total AChE activity $(B)$, and muscle weight $(C)$ in denervated SOL muscles. Similar to Figure 3 except that stimulation started after 14 days of denenvation and lasted for $6,12,24$, or $48 \mathrm{hr}$.
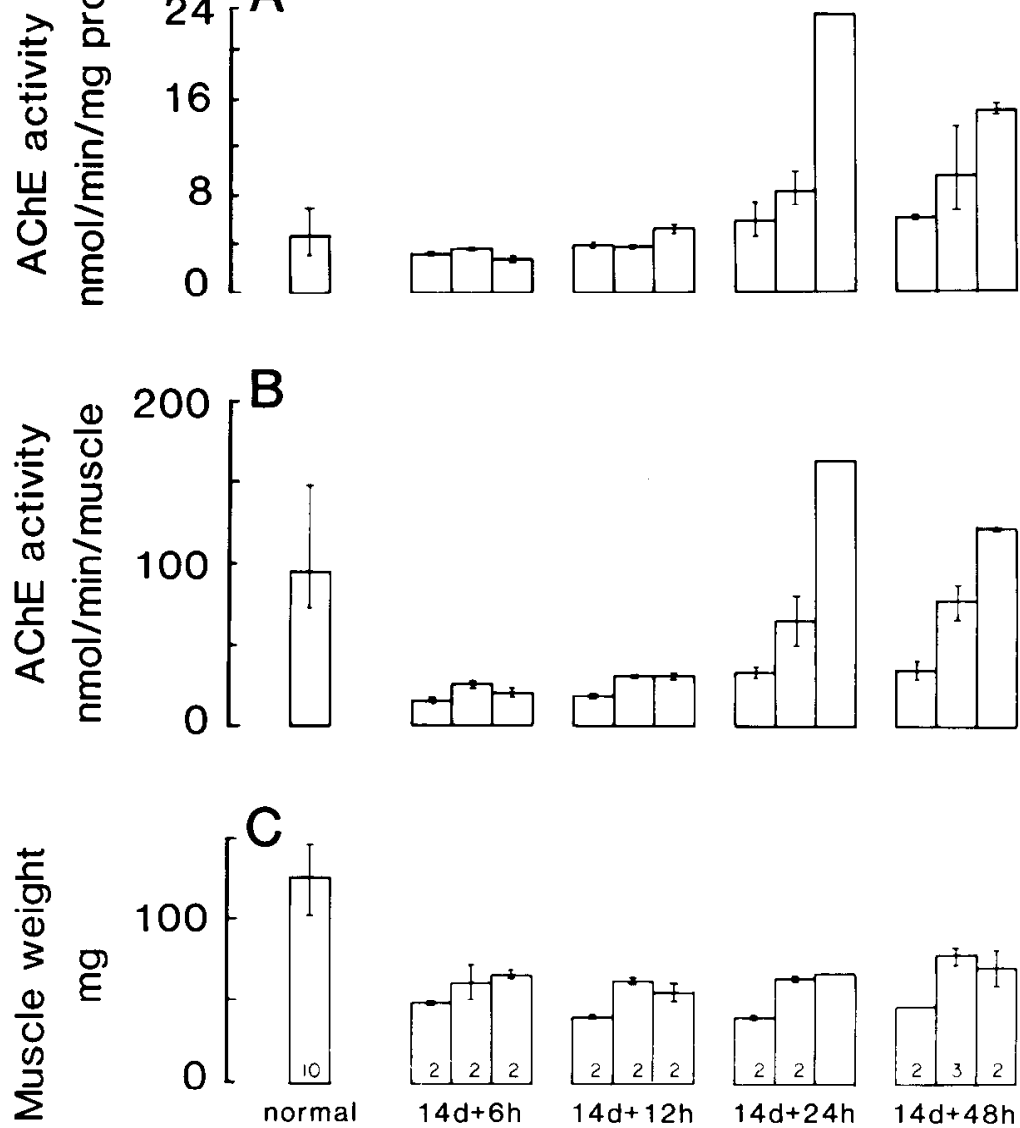

\section{Denervation $14-16$ days Stimulation last $6-48$ hours}

1 and 2) the AChE activity was examined in whole muscles, whereas in the last series (Fig. 3) it was examined in endplate-containing $(E)$ and endplate-free (NE) regions. In the first two series, 14 to 42 days of denervation reduced total $A C h E$ activity to about $25 \%$ of normal (Figs. $1 B$ and $2 B$ ). Muscle weight was similarly reduced (to about $35 \%$ of normal, Figs. $1 C$ and $2 C$ ), and the specific AChE activity was little affected (Figs. $1 A$ and $2 A$ ). In the last series, 19 to 29 days of denervation reduced not only the total, but also the specific AChE activity in endplate-free as well as endplate-containing regions (Fig. 3B). As expected from earlier studies (Hall, 1973; Vigny et al., 1976), the activity was higher in endplate-containing than in endplate-free regions of normally innervated muscles. The specific AChE activity of whole muscles appeared higher in normal EDL (Table I) than in normal SOL (Figs. 1 and 2) muscles (7.14 $\pm 0.53, n=2$ versus $4.71 \pm 0.94, n=19$; rlean $\pm \mathrm{SD}, \mathrm{nmol} / \mathrm{min} / \mathrm{mg}$ of protein). Furthermore, there was a large reduction in specific AChE activity after 13 to 16 days of denervation in EDL (Table I), whereas in the SOL the specific activity was either essentially unchanged (Figs. $1 \mathrm{~A}$ and $2 A$ ) or less markedly reduced (Fig. 3 ).

Molecular forms. Rat SOL and EDL muscles contain several AChE molecular forms in different relative proportions, as shown in Figure $4, A$ and $C$. The different forms are identified by sedimentation coefficients (Bon et al., 1979). Closely sedimenting molecules such as $G_{4}(9.9 \mathrm{~S})$ and $A_{4}(8.8 \mathrm{~S})$ partially overlap but can be safely recognized, when present in sufficient proportions.

In the normal $E D L$, the $A_{12}, G_{4}$, and $G_{1}$ forms predominate (Fig. $4 C)$. Normal SOL muscles present a more complex pattern, with significant proportions of the smaller collagen-tailed forms, $A_{8}$ and $\mathrm{A}_{4}$ (Fig. 4A). The asymmetric forms are barely detectable in nonendplate regions of two muscles (not shown). After denervation (2 to 3 weeks), they decrease markedly in SOL and EDL (Fig. 4, $B$ and $D)$. $A_{8}$ and $A_{4}$ then appear to be more abundant than $A_{12}$, and are present both in endplate and non-endplate regions (see Fig. $6, A$ and $C$ ).

Chronic stimulation of the denervated SOL muscle. In the first series of experiments on the SOL we started stimulation after 21 days of denervation and continued stimulation for 2 to 21 days. The stimulus frequency was either $10 \mathrm{~Hz}$ (slow pattern) or $100 \mathrm{~Hz}$ (fast pattern). Both patterns markedly increased specific AChE activity measured in the whole muscle (Fig. 1A). The specific activity was highest (about 3 times normal) after 2 and 7 days and then declined toward normal values during the following 2 weeks. The total AChE activity, which was very low in denervated control muscles, was transiently increased by stimulation to normal or above normal values (Fig. $1 B$ ). With continued stimulation, however, the activity fell to subnormal values, because stimulation failed to restore normal 


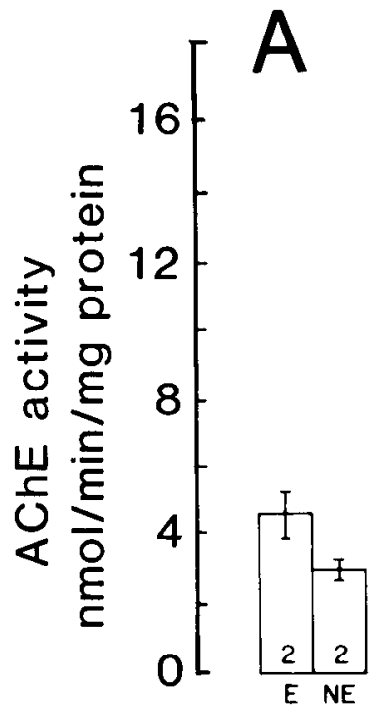

Denervated:

$-$

Stimulated:
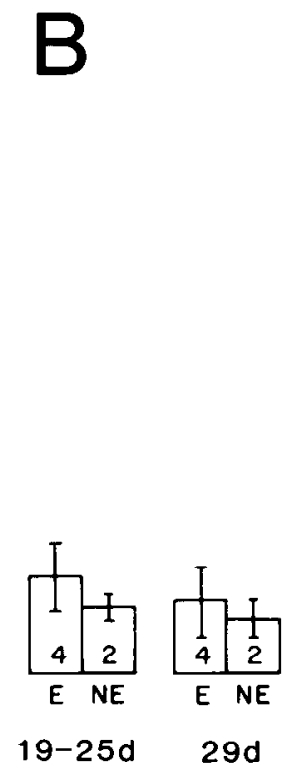

$19-25 d$
$-$

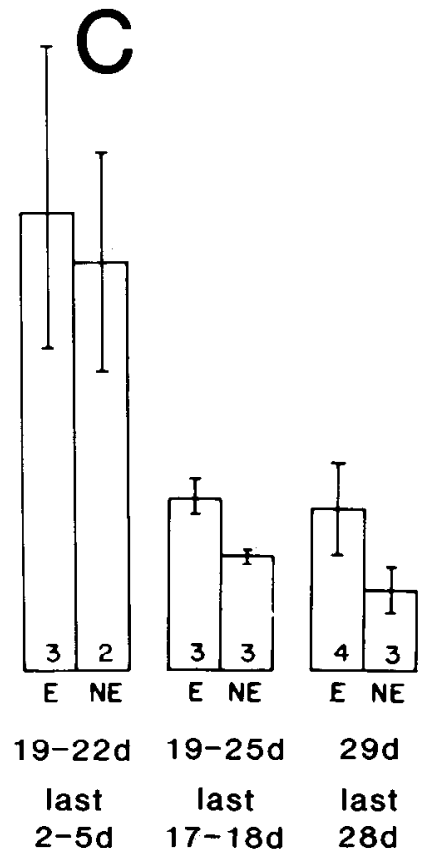

Figure 3. Specific AChE activity in endplatecontaining $(E)$ and endplate-free $(N E)$ regions of SOL muscles $(A)$ is decreased by denervation $(B)$ and increased by stimulation $(C)$. Denervation lasted 19 to 25 days and 29 days in $B$ and 19 to 22 days, 19 to 25 days, and 29 days in $C$. Stimulation in $C$ occurred during the last 2 to 5 days, 17 to 18 days, and 28 days as indicated. Each bar in $C$ represents pooled results from stimulating either intermittently at $100 \mathrm{~Hz}$ or continuously at $10 \mathrm{~Hz}$ (see "Materials and Methods"). The height of each bar indicates mean values, narrow bars give total range of all observations, and numbers in the bars give the number of muscles.
TABLE I

Effects of denervation and continuous $10 \mathrm{~Hz}$ stimulation on the $E D L$

\begin{tabular}{lccc}
\hline \multicolumn{1}{c}{ Treatment } & Muscle Weight & AChE Activity & AChE Activity \\
\hline & $m g$ & $\begin{array}{c}n \text { mol/min/ } \\
\text { muscle }\end{array}$ & $\begin{array}{c}\text { nmol/min/mg } \\
\text { of protein }\end{array}$ \\
Normal & 139,143 & 174,206 & $6.8,7.5$ \\
Denervated 13-16 days & $71.3 \pm 8.5$ & $25.5 \pm 11.6$ & $2.5 \pm 0.9$ \\
& $(n=1)$ & $(n=4)$ & $(n=4)$ \\
Denervated 16 days, & 78,89 & 32,36 & $2.8,3.0$ \\
stimulated last 2 & & & \\
days & & & \\
\hline
\end{tabular}

muscle weight. After 2 days of stimulation the denervated muscles had recovered about $40 \%$ of their previous weight loss, but continued stimulation did not further increase muscle weight (Fig. 1C).

In the second series of experiments we examined in more detail the early stimulation-induced changes in AChE activity. We now started stimulation 14 days after denervation, expecting better responsiveness at this time. However, the results were similar to those already described. In addition, they showed that both muscle weight and AChE activity began to rise as early as $12 \mathrm{hr}$ after the onset of stimulation (Fig. 2).

In the third series of experiments, some of the muscles were denervated for 3 weeks (19 to 22 days) and then stimulated for the last 2 to 5 days of this period. The stimulation causcd a marked increase in specific AChE activity to levels which were about 5 times higher than in denervated control muscles and about 3 times higher than in normal muscles (pair of bars to the left of Fig. $3 C$ ). The activity was equally high in endplate-free and endplate-containing regions, suggesting that the newly synthesized enzyme was not associated with endplates. Other SOL muscles were denervated for 19 to 29 days but stimulated during most of this time. In these muscles the specific activity was essentially normal both in regions containing the denervated endplates and outside (bars to the right of Fig. $3 C$ ). This indicates that prolonged stimulation causes a preferential increase in $\mathrm{AChE}$ activity at endplates in addition to restoring normal activities along the rest of the fiber.

The number of muscles that we have examined at each point in time is small. However, taken together, the results of the three series of experiments just described form a consistent picture which may be summarized as follows. (1) Stimulation of a 2- to 3-week dener- vated rat SOL induces a rapid transient increase in the AChE activity to several times normal values. (2) When chronic stimulation is continued for longer periods, the activity progressively returns to normal or near-normal values. (3) Initially, the stimulation appears not to affect junctional regions specifically. Later, AChE activity is preferentially localized at the denervated endplates.

\section{Different stimulus patterns have different effects on AChE molecular forms}

Two different stimulation patterns were used (intermittent $100 \mathrm{~Hz}$ and continuous $10 \mathrm{~Hz}$ ), and both patterns had similar effects on total and specific AChE activity (Figs. 1 and 2) in endplate and nonendplate regions (Fig. 3 , see legend). No consistent quantitative differences were observed, but more data are required to settle this point.

Qualitatively, however, the two patterns produced markedly different results. During intermittent $100 \mathrm{~Hz}$ stimulation (fast pattern) the proportions of the various molecular forms in denervated SOL muscles approached those in normal EDL muscles (cf. Fig. 5, $A$ to $C$ and Fig. $4 C$ ). As in the EDL, the molecular forms $A_{12}, G_{4}$, and $G_{1}$ were usually prominent, whereas the $A_{8}$ and $A_{4}$ forms normally present in the SOL were poorly represented. The increase in $A_{12}$ forms was observed both in endplate and in non-endplate regions (Fig. 6, $B$ and $D$ ).

Different results were obtained with continuous $10 \mathrm{~Hz}$ stimulation (slow pattern). After 2 to 21 days of stimulation (Fig. $5, D$ to $F$ ), the sedimentation patterns resembled those of normal SOL muscles (Fig. 4A). All globular and asymmetric forms were represented, including $A_{8}$ and $A_{4}$, normally absent in EDL. However, the relative amount of collagen-tailed forms was lower than in normal SOL muscles, but higher than in EDL. The content of $\mathrm{G}_{4}$ was also relatively high. These muscles are therefore better described as having characteristics intermediate between those in normal SOL and EDL muscles. Possible reasons for the failure of the slow stimulus pattern to maintain normal SOL properties with respect to AChE activity are discussed below.

The differential effects of the two stimulation patterns on the relative proportions of molecular AChE forms developed gradually during the first 2 days of stimulation and then remained roughly stable. It is concluded that in the denervated SOL muscle a fast stimulus pattern induces the appearance of AChE molecular forms 

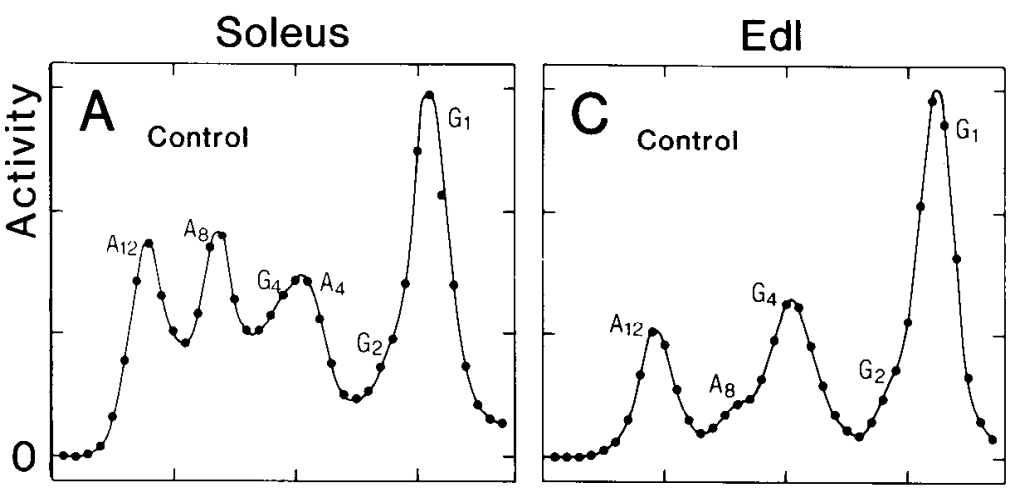

Figure 4. Different molecular forms of $\mathrm{AChE}$ in SOL $(A)$ and EDL $(C)$ muscles of rats, and effects of denervation $(B$ and $D)$. The sedimentation patterns were obtained after AChE was solubilized quantitatively by homogenizing muscle samples with 20 vol of extraction medium (see "Materials and Methods").
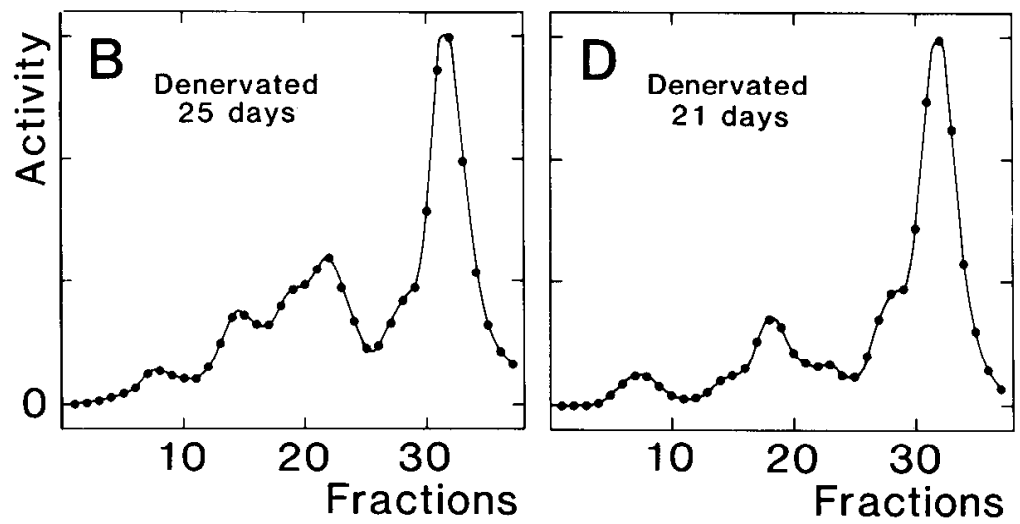

characteristic of fast muscle, whereas a slow stimulus pattern maintains the forms normally present in the soleus.

\section{Chronic stimulation of the denervated EDL}

The EDL was denervated for 14 days and then stimulated continuously for 2 days at $10 \mathrm{~Hz}$ (slow pattern). In contrast to the SOL, the EDL responded with only a small (or possibly no) increase in AChE activity (Table I). The sedimentation pattern of the different molecular forms was also apparently unaffected (data not shown). In experiments reported by Groswald and Dettbarn (1983) the specific AChE activity of the EDL remains low during early stages of reinnervation, whereas in the SOL it rises rapidly to several times normal values before it gradually declines to normal, just as it does during stimulation (see above). This suggests that EDL and SOL muscles may respond differently to activity because of intrinsic differences. However, more extensive stimulation experiments on the EDL are needed to pursue this question further.

\section{Discussion}

One main result of this work is that stimulation has a marked enhancing effect on the AChE activity of denervated rat SOL muscles. The increase was largest during the first few days of stimulation. Then followed a gradual decline in specific activity toward normal values. At least one of the stimulus patterns (fast pattern) was well within the physiological range of normal fast motor unit activity both with respect to frequency $(100 \mathrm{~Hz})$ and amount (mean frequency, 1 $\mathrm{Hz}$; Hennig and Lфmo, 1984). Therefore, these results confirm a large body of evidence (see Massoulié and Bon, 1982) that muscle activity plays an important role in the regulation of muscle AChE.

Mechanisms underlying stimulation-induced increase in AChE activity. The AChE activity of the denervated SOL started to rise 12 to $24 \mathrm{hr}$ after the onset of stimulation. This could be due to reduced release of $A C h E$ from the muscle, increased rate of synthesis, or reduced rate of $A C h E$ degradation. Denervation increases rapidly the release of $A C h E$ from rat diaphragm (Carter and Brimijoin, 1981). The possibility must therefore be considered that stimulation enhances AChE activity in the muscle by suppressing its release. Both the sarcolemma and the basal lamina are likely to be involved in
AChE release from muscle. It is therefore interesting that the properties of these structures are markedly affected by muscle activity (Lфmo and Westgaard, 1976; Sanes and Lawrence, 1983). Muscle activity probably stimulates AChE synthesis. From a low basal level of synthcsis in completely inactive muscle cultures, fibrillatory activity increases $A C h E$ activity primarily through an effect on synthesis (Brockman et al., 1984). The regulation of AChE levels may also involve changes in the degradation rate. For example in cultures of a murine cell line, T 28, and in primary cultures of avian muscle cells, only a minor fraction of rapidly degraded AChE monomers or inactive precursors reaches the external cellular surface or is secreted in an active form (Lazer et al., 1984; R. L. Rotundo, personal communication). Finally, denervation activates proteases in muscle (McLaughlin et al., 1974; Fernandez and Duell, 1980), and this could reducc $A C h E$ activity either by increasing the rate of degradation or by affecting the surface membranes of the muscle so that more $A C h E$ is released.

Regulation of AChE in junctional and extrajunctional regions. The specific AChE activity was higher in endplate-containing than in endplate-free regions of innervated SOL muscles in agreement with the high concentration of AChE at neuromuscular junctions (Hall, 1973; Vigny et al., 1976). Denervation reduced AChE activity, whereas stimulation substantially increased it, initially to the same degree in both endplate-containing and endplate-free regions. Later, when the specific activity declined toward normal values, the normal difference betwen the two regions was re-established (Fig. 3). The simplest explanation of these results is that muscle activity quickly induces the appearance of AChE all long the fiber. Then, after a delay, $A C h E$ begins to accumulate at the denervated endplates, owing to earlier local instructions laid down by the nerve. Present and earlier evidence ( $L$ $\phi \mathrm{mo}$ and Slater, 1980) suggest that such "instructions" remain for at least some weeks after removal of the nerve. It is not known, however, how long they can be maintained. These results strengthen the conclusion that muscle activity may be responsible for the normalizing effect of ectopic innervation on the AChE content of denervated endplates (Weinberg and Hall, 1979).

Clearly, neural influences other than evoked muscle activity are responsible for the precise localization of AChE at neuromuscular 


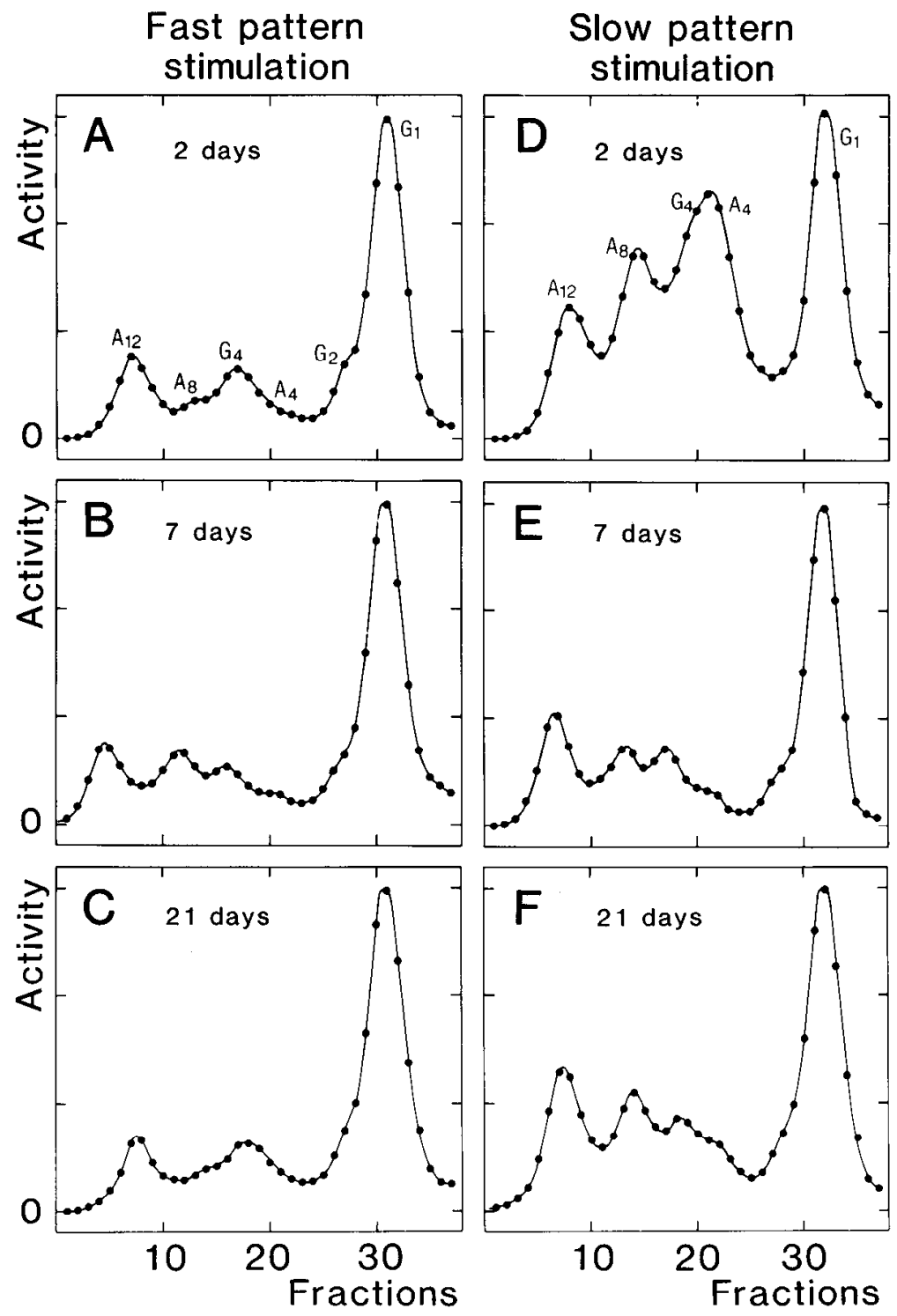

Figure 5. Stimulus pattern influences the distribution of AChE molecular forms in denervated SOL muscies. Stimulation was intermittent at $100 \mathrm{~Hz}$ in $A$ to $C$ and continuous at $10 \mathrm{~Hz}$ in $D$ to $F$. Durations of denervation and of stimulation were, respectively, 23 days and the last 2 days in $A$ and $D, 22$ days and the last 7 days in $B$ and $E$, and 22 days and the last 21 days in $C$ and $F$.

junctions. Several lines of evidence indicate that neural substances cooperate with evoked muscle activity in the regulation of junctional properties, particularly AChE. Substances present in extracts from neural tissues partially prevent the loss of AChE activity at denervated endplates (Davey et al., 1979; Fernandez et al., 1980) and also induce AChR clustering in noninnervated muscle fibers (Jessel et al., 1979). However, normal maturation of motor endplates, including the appearance of postjunctional folds, AChR channel complexes with short open times, and $\mathrm{AChE}$, appears to require evoked muscle activity (Brenner et al., 1983; L $\phi$ mo et al., 1984b).

The function and regulation of $A C h E$ in extrajunctional regions are less clear. In some muscles extrajunctional AChE activity decreases after denervation (Fig. 3) whereas, in others, it increases (Bacou et al., 1982). Regardless of the direction of the response to denervation, however, evoked muscle activity returns the AChE activity of noninnervated muscles toward normal values (Fig. 3; Walker and Wilson, 1975). Nerve extracts do not appear to influence extrajunctional AChE activity in denervated muscle (Davey et al., 1979). Furthermorc, therc is an apparent dissociation between the regulation of $A C h E$ and $A C h$ receptors (AChRs) in extrajunctional regions since denervation stimulates the synthesis and appearance of AChRs regardless of whether the AChE activity simultaneously increases or decreases. In some muscles, the $A_{12}$ molecular form of AChE is located highly concentrated at the endplate, suggesting that it may have a specific role in nerve-muscle interaction. In the rat SOL, however, this form is detected also outside the endplate even after several weeks of denervation and stimulation increases its activity both in endplate-containing and endplate-free regions. It appears from these varied observations that evoked muscle activity plays an important role in the regulation of both extrajunctional and junctional AChE. In the extrajunctional region, however, certain features of the regulation depend dramatically on the species and type of muscle. Moreover, in this region there is, so far, little evidence that activityindependent neurotrophic influences play an important role.

Significance of pattern of muscle activity. A second main result of this work is that stimulation affects the distribution of $\mathrm{AChE}$ molecular forms in the SOL in a stimulus pattern-dependent manner. First, it is noteworthy, however, that regardless of the pattern, stimulation increased the relative proportion of the A-forms, providing new evidence of the muscular origin of these forms and of the capacity of the muscle to synthesize the A-forms in the absence of the nerve. The fast pattern resulted in a distribution of AChE molecular forms that was similar to that in normal EDL muscles: the $G_{1}$, $G_{4}$, and $A_{12}$ forms were prominent components, whereas $A_{4}$ and $A_{8}$ were only present in minor proportions. The slow pattern, on the other hand, did not suppress the $A_{8}$ and $A_{4}$ forms but produced a sedimentation pattern similar but not identical to that of normal slow SOL muscles. The failure to recover a completely normal SOL pattern may be due to the particular stimulation pattern used. We now know that normal SOL motor units discharge around 18 to $20 \mathrm{~Hz}$ (and not 
Figure 6. Denervation decreases ( $A$ and $C$ ) and stimulation increases $(B$ and $D)$ the content of asymmetric AChE molecular forms, especially the $A_{12}$ form, in endplate-free $(A$ and $B)$ and endplate-containing $(C$ and $D)$ regions of SOL muscles. The sedimentation patterns were obtained from two 23-day denervated SOL muscles from one rat. One muscle had been stimulated intermittently at $100 \mathrm{~Hz}$ for the last 5 days.

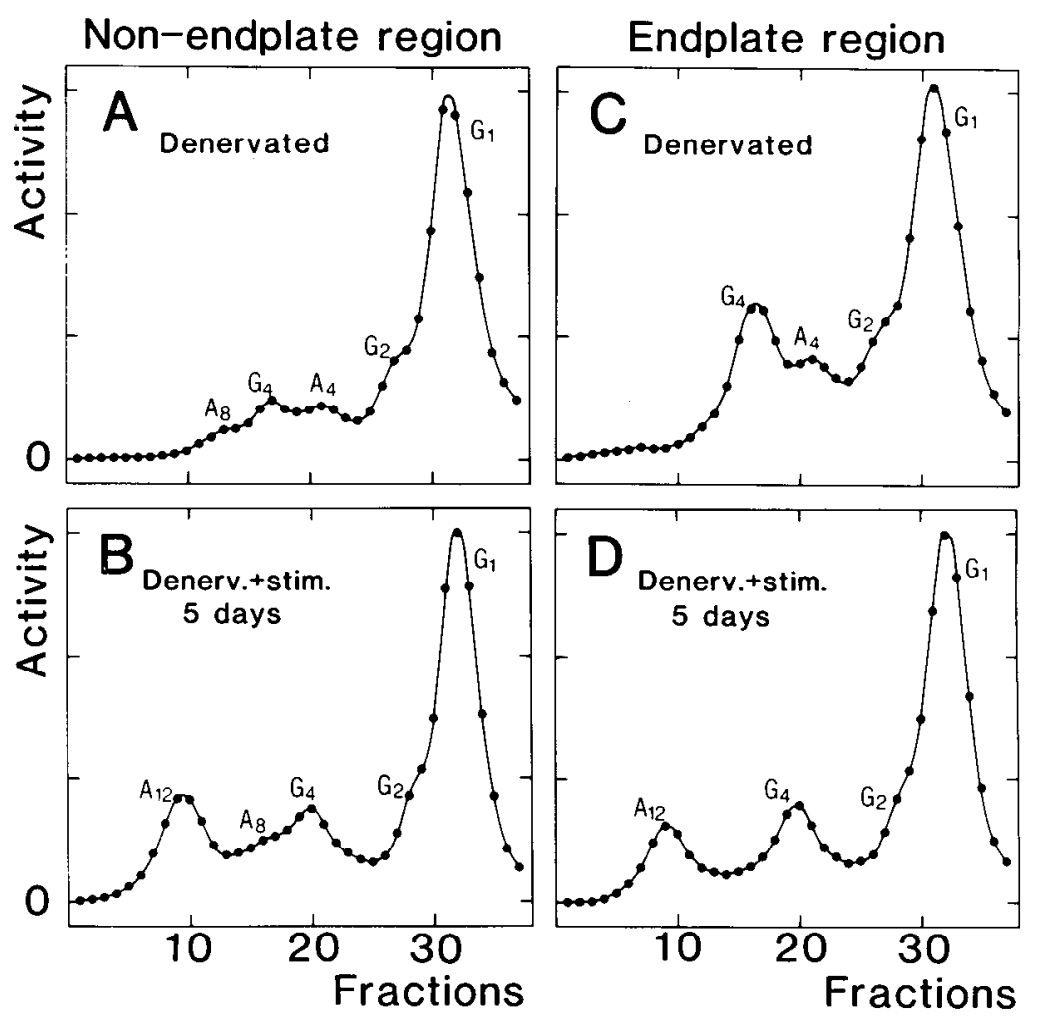

$10 \mathrm{~Hz}$ ) and are intermittently active for about 20 to $35 \%$ of the time, rather than being continuously active (Hennig and $L \phi m o, 1984$ ). The answer to whether chronic stimulation can maintain a normal distribution of AChE molecular forms in the denervated SOL must therefore await stimulation with patterns more typical of normal SOL motor units.

The functional significance of the different AChE molecular forms is not known. One possibility is that effective neuromuscular transmission requires different junctional properties depending upon the rate of impulses arriving at the junction. In normal rat SOL and EDL motor units, the impulse rates vary around 20 and 50 to $90 \mathrm{~Hz}$, respectively (Hennig and $L \phi m o, 1984$ ). This could explain why motor endplates in fast muscles, such as the EDL, have higher AChR densities (Sterz et al., 1983), deeper and more extensive postjunctionial folds (Padykula and Gauthier, 1970), higher AChE activity (this work), and different distributions of AChE molecular forms (Fig. 4) compared to the endplates in slow muscles.

Although the catalytic activity of the different AChE molecular forms appears to be the same, their physical properties differ and this may well influence their localization and hence their function at the motor endplate (Vigny et al., 1978). If effective transmission of fast and slow impulse rates requires different junctional properties, then the cellular processes determining these properties are likely to be modified by changes in impulse rates. It has been shown elsewhere that the twitch contraction speed of denervated rat SOL muscles becomes much faster during direct stimulation with a fast stimulus pattern, whereas a slow contraction speed is maintained during stimulation with a slow pattern. As a result, effective rate modulation of muscle force output can be maintained despite even large changes in imposed impulse patterns (L $\phi$ mo et al., 1984a). The present experiments indicate that the different AChE molecular forms at the neuromuscular junction also adapt to varying impulse patterns.

\section{References}

Bacou, F., P. Vigneron, and J. Massoulié (1982) Acetylcholinesterase forms in fast and slow rabbit muscle. Nature 296: 661-664.

Bon, S., M. Vigny, and J. Massoulié (1979) Asymmetric and globular forms of acetylcholinesterase in mammals and birds. Proc. Natl. Acad. Sci. U. S. A. 76 : $2546-2550$

Brenner, H. R., T. Meier, and B. Widmer (1983) Functional and structural differentiation of subsynaptic membrane in rat muscle denervated at early stages of endplate development. Nature 305: 536-537.

Brockman, S. K., L. H. Younkin, and S. G. Younkin (1984) The effect of spontaneous electromechanical activity on the metabolism of acetylcholinesterase in cultured embryonic rat myotubes. J. Neurosci. 4: 131-141.

Butler, I. J., D. B. Drachman, and A. M. Goldberg (1978) The effect of disuse on cholinergic enzymes. J. Physiol. (Lond.) 274: 593-600.

Cangiano, A., T. Lфmo, L. Lutzemberger, and O. Sveen (1980) Effects of chronic nerve conduction block on formation of neuromuscular junctions and junctional AChE in the rat. Acta Physiol. Scand. 109: 283-296.

Carson, S., S. Bon, M. Vigny, J. Massoulié, and M. Fardeau (1979) Distribution of acetylcholinesterase molecular forms in neural and non-neural sections of human muscle. FEBS Lett. 97: 348-352.

Carter, J. L., and S. Brimijoin (1981) Effects of acute and chronic denervation on release of acetylcholinesterase and its molecular forms in rat diaphragms. J. Neurochem. 36: 1018-1025.

Collins, P., and S. G. Younkin (1982) Effect of denervation on the molecuiar forms of acetylcholinesterase in rat diaphragm. J. Biol. Chem. 257: 1363813644

Davey, B., L. H. Younkin, and S. G. Younkin (1979) Neural control of skeletal muscle cholinesterase: A study using organ-cultured rat muscle. J. Physiol. (Lond.) 289: 501-515.

Drachman, D. B. (1972) Neurotrophic regulation of muscle cholinesterase: Effects of botulinum toxin and denervation. J. Physiol. (Lond.) 226: 619627.

Ellman, G. L., K. D. Courtney, V. Andres, Jr., and R. M. Featherstone (1961) A new and rapid colorimetric determination of acetylcholinesterase activity. Biochem. Pharmacol. 7: 88-95.

Fernandez, H. L., and M. J. Duell (1980) Protease inhibitors reduce effects of denervation on muscle endplate acetylcholinesterase. J. Neurochem. 35: 1166-1171.

Fernandez, H. L., M. R. Patterson, and M. J. Duell (1980) Neurotrophic control of $16 \mathrm{~S}$ acetylcholinesterase tor mammalian skeletal muscle in organ culture. J. Neurobiol. 11: 557-570.

Fischbach, G. D., and N. Robbins (1969) Changes in contractile properties of disused soleus muscles. J. Physiol. (Lond.) 201: 305-320.

Gisiger, V., and H. Stephens (1982) Acetylcholinesterase content in both motor nerve and muscle is correlated with twitch properties. Neurosci. Lett. 31: 301-305. 
Groswald, D. E., and W. -D. Dettbarn (1983) Nerve crush induced changes in molccular forms of acetylcholinesterase in soleus and extensor digitorum muscles. Exp. Neurol. 79: 519-531.

Hall, Z. W. (1973) Multiple forms of acetylcholinesterase and their distribution in endplate and non-endplale regions of ral diaphragm muscle. J. Neurobiol. 4: 343-361.

Hennig, R., and T. Lфmo (1984) Discharge patterns of presumed FF, FR and $\mathrm{S}$ motor units during normal motor behaviour in the rat. Acta Physiol. Scand. 121: $28 \mathrm{~A}$.

Jessel, T. M., R. E. Siegel, and G. D. Fischbach (1979) Induction of acetylcholine receptors on cultured skeletal muscle by a factor extracted from brain and spinal cord. Proc. Natl. Acad. Sci. U. S. A. 76: 5397-5401.

Katz, B., and R. Miledi (1973) The binding of acetylcholine to receptors and its removal from the synaptic cleft. J. Physiol. (Lond.) 231: 549-574.

Koenig, J., and F. Rieger (1981) Biochemical stability of the AChE molecular forms after cytochemical staining: Post-natal focalization of the high molecular weight forms of rat AChE. Dev. Neurosci. 4: 249-257.

Koenig, J., and M. Vigny (1978) Neural induction of the $16 \mathrm{~S}$ acetylcholinesterase in muscle cell cultures. Nature 271: 75-77.

Lazar, M., E. Salmeron, M. Vigny, and J. Massoulié (1984) Heavy isotope labeling study of the metabolism of monomeric and tetrameric acetylcholinesterase in the murine neuronal-like $T_{28}$ hybrid cell line. J. Biol. Chem. 259: 3703-3713.

Lфmo, T., and C. R. Slater (1980) Control of junctional acetylcholinesterase by neural and muscular influences in the rat. J. Physiol. (Lond.) 303: $191-$ 202.

Lomo, T., and R. H. Westgaard (1976) Control of ACh sensitivity in rat muscle fibres. Cold Spring Harbor Symp. Quant. Biol. 40: 263-274.

Lфmo, T., R. H. Westgaard, and H. A. Dahl (1974) Contractile properties of muscle: Control by pattern of muscle activity in the rat. Proc. R. Soc. Lond. (Biol.) 187: 99-103

Lфmo, T., K. Gundersen, R. Hennig, and R. H. Westgaard (1984a) Maintenance and regulation of contractile properties by chronic stimulation of denervated muscles in the rat: Role of impulse patterns. In Recent Achievements in Restorative Neurology: Upper Motor Neurone Functions and Dysfunctions, J. C. Eccles and M. R. Dimitrijevic, eds., S. Karger AG, Basel, in press.

Lфmo, T., R. Mirsky, and S. Pockett (1984b) Formation of neuromuscular junctions in adult rats: Role of postsynaptic impulse activity. In Neuromuscular Disease, G. Serratrice, D. Cros, C. Desnuelle, J. -L. Gastaut, J. -F.
Pellisier, J. Pouget, and A. Schiano, eds., pp. 393-399, Raven Press, New York.

Lowry, O. H., N. J. Rosebrough, A. L. Farr, and R. J. Randall (1951) Protein measurement with the Folin phenol reagent. J. Biol. Chem. 193: 265-275.

Massoulié, J., and S. Bon (1982) The molecular forms of cholinesterase and acetylcholinesterase in vertebrates. Annu. Rev. Neurosci. 5: 57-106.

McLaughlin, J., L. G. Abood, and H. B. Bosmann (1974) Early elevations of glycosidase, acid phosphatase, and acid proteolytic activity in denervated skeletal muscle. Exp. Neurol. 42: 541-554.

Padykula, H. A., and G. F. Gauthier (1970) The ultrastructure of the neuromuscular junctions of mammalian red, white and intermediate skeletal muscle fibers. J. Cell. Biol. 46: 27-41.

Rieger, F., J. Koenig, and M. Vigny (1980) Spontaneous contractile activity and the presence of the $16 \mathrm{~S}$ form of acetylcholinesterase in rat muscle cells in culture. Reversible suppressive action of tetrodotoxin. Dev. Biol. 76: 358-365.

Rubin, L. L., S. M. Schuetze, C. L. Weill, and G. D. Fischbach (1980) Regulation of acetylcholinesterase appearance at neuromuscular junctions in vitro. Nature 283: 264-267

Salmons, S., and F. A. Sreter (1976) Significance of impulse activity in the transformation of skeletal muscle type. Nature 263: 30-34.

Sanes, J. R., and J. C. Lawrence, Jr. (1983) Activity-dependent accumulation of basal lamina by cultural rat myotubes. Dev. Biol. 97: 123-136.

Sketelj, J., and M. Brzin (1980) $16 \mathrm{~S}$ acetylcholinesterase in endplate-free regions of developing rat diaphragm. Neurochem. Res. 5: 655-660.

Sterz, R., M. Pagala, and K. Peper (1983) Postjunctional characteristics of the endplates in mammalian fast and slow muscles. Pflügers Arch. 398: 48-54.

Vigny, M., J. Koenig, and F. Rieger (1976) The motor end-plate specific form of acetycholinesterase: Appearance during embryogenesis and re-innervation of rat muscle. J. Neurochem. 27: 1347-1353.

Vigny, M., S. Bon, J. Massoulié, and F. Leterrier (1978) Active site catalytic efficiency of acetylcholinesterase molecular forms in Electrophorus, Torpedo, rat and chicken. Eur. J. Biochem. 85: 317-323.

Walker, C. R., and B. W. Wilson (1975) Control of acetylcholinesterase by contractile activity of cultured muscle cells. Nature 256 : 215-216.

Weinberg, C. B., and Z. W. Hall (1979) Junctional form of acetylcholinesterase restored at nerve-free endplates. Dev. Biol. 68: 631-635.

Younkin, S. C. Rosenstein, P. L. Collins, and T. L. Rosenberry (1982) Cellular localization of the molecular forms of acetylcholinesterase in rat diaphragm. J. Biol. Chem. 257: 13630-13637. 